Pacific Journal of Mathematics

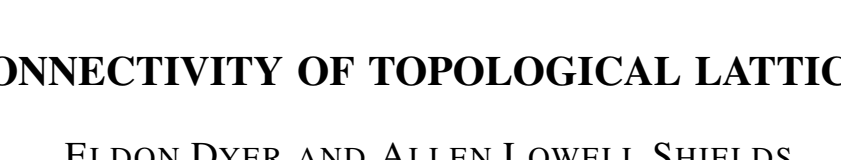




\section{CONNECTIVITY OF TOPOLOGICAL LATTICES}

\section{Eldon Dyer ANd Allen Shields}

In this note we show that compact connected topological lattices have strong acyclicity properties, both globally and locally. This yields a proof of a conjecture of A. D. Wallace [6] in the finite dimensional case.

A topological lattice is a topological space (Hausdorff) upon which is imposed a lattice structure compatible with the topology. More explicitly, the topological space $M$ is a topological lattice if there are maps

$$
\Lambda: M \times M \rightarrow M \text { and } \mathrm{V}: M \times M \rightarrow M
$$

which define a lattice structure on $M$. This means that for $x, y, z \in M$

$$
\begin{aligned}
& \Lambda(x, x)=x \text { and } \mathrm{V}(x, x)=x \\
& \Lambda(x, y)=\Lambda(y, x) \text { and } \mathrm{V}(x, y)=\mathrm{V}(y, x) \\
& \Lambda(x, \Lambda(y, z))=\Lambda(\Lambda(x, y), z) \text { and } \\
& \mathrm{\vee}(x, \mathrm{~V}(y, z))=\mathrm{V}(\mathrm{\vee}(x, y), z), \text { and }
\end{aligned}
$$

It is customary to write $x \wedge y$ in place of $\Lambda(x, y)$ and $x \vee y$ in place of $\mathrm{V}(x, y)$. Relation (5) implies that $x \wedge y=x$ if and only if $x \vee y=y$. We shall say that $x \leqq y$ if and only if $x \wedge y=x$. It is easily seen that the relation $x \leqq y$ induces a partial ordering on $M$. The element $1 \in M$ is a unit in $M$ provided $m \leqq 1$ for all $m \in M$. Similarly, an element $0 \in M$ is a zero in $M$ if $0 \leqq m$ for all $m \in M$. Clearly, if such elements exist they are unique.

We shall need several elementary lemmas on topological lattices. Lemmas 2 and 4 were proved in [1], however for completeness we prove them here. Lemma 1 was proven by A. D. Wallace [7].

LEMMA 1. If $M$ is a compact topological lattice, then it has a unit and a zero.

Lemma 2. If $M$ is a topological lattice, then

(a) if $U$ is a neighborhood of $x \in M$, there is a neighborhood $V$ of $x$ such that if $y, z \in V$, then $y \vee z \in U$ and $y \wedge z \in U$, and

(b) if $y \leqq x$ and $U_{x}$ is a neighborhood of $x$, there are neighbor- 
hoods $V_{x}$ of $x$ and $V_{y}$ of $y$ such that if $x^{\prime} \in V_{x}$ and $y^{\prime} \in V_{y}$, then $x^{\prime} \vee y^{\prime} \in U_{x}$.

To prove (a) we observe that $\Lambda^{-1}(U) \cap \mathrm{V}^{-1}(U)$ is a neighborhood of $(x, x) \in M \times M$ and that there there is a neighborhood $V$ of $x$ such that $V \times V \subset \wedge^{-1}(U) \cap \mathrm{V}^{-1}(U)$. Then if $y$ and $z$ lie in $V,(y, z)$ lies in this intersection, and so $y \vee z \in U, y \wedge z \in U$.

In (b) $\mathrm{V}^{-1}\left(U_{x}\right)$ is a neighborhood of $(x, y) \in M \times M$ and there are neighborhoods $V_{x} \times V_{y} \subset \mathbf{V}^{-1}\left(U_{x}\right)$. Clearly these neighborhoods have the asserted properties.

Lemma 3. If $M$ is a compact topological lattice and $U$ is a neighborhood of $x \in M$, there is a neighborhood $W$ of $x$ such that if $y, z \in W$ and $m \in M$, then $(m \wedge y) \vee z \in U$.

For $m \in M$ let $V(m)$ and $N(m)$ be neighborhoods of $x$ and $x \wedge m$ as in Lemma 2(b). Let $P(m)$ and $Q(m)$ be neighborhoods of $m$ and $x$ such that $P(m) \times Q(m) \subset \Lambda^{-1}(N(m))$ and let $R(m)=V(m) \cap Q(m)$. Then if $m^{\prime} \in P(m)$ and $y, z \in R(m), m^{\prime} \wedge y \in N(m)$ and $\left(m^{\prime} \wedge y\right) \vee z \in U$. Since $M$ is compact, there is a finite set $\left[m_{i}\right]_{1}^{n}$ of points of $M$ such that $\bigcup_{1}^{n} P\left(m_{i}\right)=$ $M$. Let $W=\bigcap_{1}^{n} R\left(m_{i}\right)$. Then $W$ is the required neighborhood of $x$.

For $x, y \in M, x \leqq y$, we define

$$
C_{x, y}=\{z \in M \mid x \leqq z \leqq y\} .
$$

Such sets $C_{x, y}$ will be called convex sets. It is clear that if $M$ is a compact topological lattice, then its convex subsets are also compact topological lattices in their natural lattice and topological structures.

Lemma 4. If $M$ is a compact topological lattice and $U$ is a neighborhood of $x$, then there is a nighborhood $V$ of $x$ such that if $y \in V$, then $C_{y, y \vee x} \cup C_{x, y \vee x} \subset U$.

Let $W$ be a neighborhood of $x$ as in Lemma 3 and $V$ be a neighborhood of $x$ for $W$ as in Lemma 2(a). Then if $y \in V, y \vee x \in W$. If $y \leqq$ $z \leqq y \vee x$, then $z=(z \wedge\{y \vee x\}) \vee y \in U$ and if $x \leqq z \leqq y \vee x$, then $z=$ $(z \wedge\{y \vee x\}) \vee x \in U$.

A space $X$ is acyclic if $H^{*}(X)=0$, where $H^{*}()$ denotes the reduced cohomology ring; $X$ is clc if for each $x \in X$ and closed neighborhood $U$ of $x$, there is a closed neighborhood $V$ of $x, V \subset U$, such that the homomorphism of reduced groups $H^{*}(U) \rightarrow H^{*}(V)$ induced by inclusion is trivial.

Before proceding to the proof of Theorem 1 we recall a well-known generalization of the fact that homotopic maps induce the same homomorphisms of cohomology. 
Theorem. If $X$ and $Y$ are compact, $N$ is compact and connected, $n_{1}, n_{2}$ are two points of $N, f, g: X \rightarrow Y$, and $F: X \times N \rightarrow Y$ are given such that $F \mid X \times n_{1}=f$ and $F \mid X \times n_{2}=g$, then $f^{*}=g^{*}: H^{*}(Y) \rightarrow H^{*}(X)$.

THEOREM 1. If the compact topological lattice $M$ is connected, then it is acyclic and cle.

The fact that $M$ is acyclic was first proven by A. D. Wallace [8]. We give here a slightly different proof.

Let $\hat{M}=(M \times 1) \cup(1 \times M) \subset M \times M$. Since $M$ is connected and $1 \times 1 \in(M \times 1) \cap(1 \times M), \hat{M}$ is connected. For $x, y \in M$ define

$$
f_{x, y}: \hat{M} \rightarrow C_{y, y \vee x} \cup C_{x, y \vee x}
$$

by

$$
f_{x, y}(m, 1)=(m \wedge\{y \vee x\}) \vee y \text { and } f_{x, y}(1, m)=(m \wedge\{y \vee x\}) \vee x .
$$

Note that

$$
(1 \wedge\{y \vee x\}) \vee y=y \vee x=(1 \wedge\{y \vee x\}) \vee x .
$$

Define $G: M \times \hat{M} \rightarrow M$ by $G(m, \hat{m})=f_{0, m}(\hat{m})$. Then

$$
G(m,(0,1))=f_{0, m}(0,1)=(0 \wedge\{m \vee 0\}) \vee m=m
$$

and

$$
G(m,(1,0))=f_{0, m}(1,0)=(0 \vee\{m \vee 0\}) \wedge 0=0 .
$$

If $i: M \rightarrow M$ is the identity and $j: M \rightarrow 0 \stackrel{\ulcorner}{\rightarrow} M$, then $i=G \mid M \times(0,1)$ and $j=G \mid M \times(1,0)$. Hence $i^{*}=j^{*}$. But $i^{*}$ is the identity isomorphism of $H^{*}(M)$, and $j^{*}$ is trivial. Hence, $M$ is acyclic.

For a closed neighborhood $U$ of $x \in M$, let $V$ be a closed neighborhood of $x$ as in Lemma 4. Define $F: V \times \hat{M} \rightarrow U$ by

$$
F(v, \hat{m})=f_{x, v}(\hat{m}) \subset C_{v, v \vee x} \cup C_{x, v \vee x} \subset U .
$$

Note that $F \mid V \times(0,1)$ is the inclusion map of $V$ into $U$ and that $F \mid V \times(0,1)$ is the trivial map of $V$ onto $x$. It follows as before that the inclusion map induces the trivial cohomology homomorphism, and hence, that $M$ is clc.

In this connection we remark that Lee Anderson [1] has shown that a locally compact connected lattice is locally connected.

An immediate consequence of Theorem 1 and results of E. G. Begle [2] is the following. 
Corollary 1. If $M$ is a finite dimensional, compact connected topological lattice, then $M$ has the fixed point property.

A slightly stronger statement is also true; namely, if $M$ is such a lattice and $f$ is an upper semi-continuous mapping of $M$ into the set of its convex subsets, then some element of $M$ lies in its image.

THEOREM 2. If the compact metric topological lattice $M$ is connected, then it is contractible and locally contractible.

Since $M$ is $\mathrm{clc}^{\circ}$, it is locally connected. Thus, $\hat{M}$ is a compact, connected, locally connected metric space. It follows that there is a mapping $h: I \rightarrow \hat{M}$ such that $h(0)=(0,1) \in \hat{M}$ and $h(1)=(1,0) \in \hat{M}$. Here $I$ denotes the unit interval.

Define $H: M \times I \rightarrow M$ by $H(m, t)=G(m, h)$. Then $H$ is the contracting homotopy sought. For $V \subset U$ as in the proof of Theorem 1, define $J: V \times I \rightarrow U$ by $J(v, t)=F(v, h(t))$. Then $J$ is a contraction of $V$ to $x$ within $U$.

A consequence of this theorem and standard results on absolute neighborhood retracts (see, for example, [5] Propositions 12.2b, 16.4, 19.2) is the following.

Corollary 2. If $M$ is a finite dimensional, compact metric, connected topological lattice, then it is an absolute retract.

Any convex subset of a compact connected topological lattice has these same properties itself, and is thus acyclic and clc. Furthermore, the intersection of finitely many convex subsets is a convex subset. We shall show that if $M$ satisfies certain additional conditions, it has a neighborhood basis of convex subsets.

A lattice $M$ is said to be distributive if for $x, y, z \in M$

$$
(x \vee y) \wedge z=(x \wedge z) \vee(y \wedge z) .
$$

A lattice $M$ is said to be of breadth $b$ if for each finite set [x] of more than $b$ elements of $M$, there is a subset $\left[y_{i}\right]$ of $b$ elements such that $x_{1} \wedge x_{2} \wedge \cdots=y_{1} \wedge y_{2} \wedge \cdots \wedge y_{b}$, and $b$ is the least number for which this holds. Similarly, one can define the breadth, $b_{1}$, using joins instead of meets. It is then a simple fact that $b=b_{1}$ (see [3] p. 20).

THEOREM 3. If $M$ is a compact distributive topological lattice of finite breadth and $U$ is a neighborhood of a point $x \in M$, then there is a convex set $C_{y, z}$ that is a neighborhood of $x$ and lies in $U$. 
Let $b$ denote the breadth of $M$, let $W_{1}$ denote a closed neighborhood of $x$ as in Lemma 3 for the neighborhood $U$, and let $W_{2}, \cdots, W_{2 b}$ denote neighborhoods of $x$ such that for $2 \leqq i \leqq 2 b$, if $y, z \in W_{i}$ then $y \vee z \in W_{i-1}$ and $y \wedge z \in W_{i-1}$. Let $R$ denote the union of the ranges of all lattice polynomials over the domain $W_{2 b}$. By Theorem 12, p. 145 of [3], any such polynomial can be written in the form

$$
\bigwedge_{n=1}^{r}\left[\bigvee_{k=1}^{n(n)} x_{i(h, k)}\right]
$$

Since each $\mathrm{V} x_{i(h, k)}$ is the join of not more than $b$ elements of $W_{2 b}$, every such element lies in $W_{b}$. Hence, any element in the range of a lattice polynomial over $W_{2 b}$ is the meet of not more than $b$ elements of $W_{b}$ and so lies in $W_{1}$. Thus, $R \subset W_{1}$. Since $R$ is a sublattice of $M$, the closure $\bar{R}$ of $R$ is a sublattice of $M$, and $\bar{R} \subset W_{1} . \quad \bar{R}$ is a compact topological lattice and by Lemma 1 has a unit $a$ and a zero $b$. By Lemma 3, $C_{b, a} \subset U . \quad C_{b, a}$ is a neighborhood of $x$ since $W_{2 b} \subset R \subset \bar{R} \subset C_{b, a}$.

In closing we would like to note the following conjectures.

Suppose $M$ is a compact, metric, connected, distributive topological lattice. Then

(i) $M$ admits sufficiently many lattice homomorphisms onto the unit interval to separate points;

(ii) $M$ is an absolute retract;

(iii) $\operatorname{dim} M=$ breadth of $M$; and

(iv) if $\operatorname{dim} M=n, M$ is homeomorphic to a subset of an $n$-cell.

D. E. Edmondson has announced [4] an example of a compact, metric connected two dimensional lattice that is modular but not distributive, and that cannot be imbedded in the plane. Lee Anderson has a proof (unpublished) that breadth $M \leqq \operatorname{dim} M$. Therefore in Theorem 3 the hypothesis that $M$ has finite breadth may be replaced by the hypothesis that $M$ is finite dimensional.

\section{REFERENCES}

1. Lee Anderson, Topological lattices, Tulane University dissertation 1956, to appear, Trans. Amer. Math. Soc.

2. E. G. Begle, A fixed point theorem, Ann. of Math., 51 (1950), 544-550.

3. Garrett Birkhoff, Lattice theory, Amer. Math. Soc. Colloquium Publications, 25, New York 1948.

4. D. E. Edmondson, A modular topological lattice, Abstract 540-10, Notices, Amer. Math. Soc., 5 (1958), No. 1, p. 92.

5. Solomon Lefschetz, Topics in topology, Ann. of Math. Studies, 10, Princeton University press, 1942.

6. A. D. Wallace, Research Problem No. 9, Bull. Amer. Math. Soc., 61 (1955), 93. 
7. A note on mols, Anais Acad. Brasil. Ci. 24 (1952), 329-334.

8. Cohomology, dimension and mobs, Summa Brasiliensis Math. 3 (1953), 43-55.

\section{UNIVERSITY OF CHICAGO}

AND

UNIVERSITY OF MICHIGAN 


\section{PACIFIC JOURNAL OF MATHEMATICS}

\section{EDITORS}

\section{David Gilbarg}

Stanford University

Stanford, California

\section{R. A. Beaumont}

University of Washington

Seattle 5 , Washington

\author{
A. L. Whiteman
}

University of Southern California Los Angeles 7, California

L. J. Paige

University of California

Los Angeles 24, California

\author{
E. F. BECKENBACH \\ C. E. BURGESS \\ E. HEWITT \\ A. HORN
}

\author{
V. GANAPATHY IYER \\ R. D. JAMES \\ M. S. KNEBELMAN \\ L. NACHBIN
}

ASSOCIATE EDITORS
I. NIVEN

T. G. OSTROM

H. L. ROYDEN

M. M. SCHIFFER
E. G. STRAUS

G. SZEKERES

F. WOLF

K. YOSIDA

\section{SUPPORTING INSTITUTIONS}

\author{
UNIVERSITY OF BRITISH COLUMBIA \\ CALIFORNIA INSTITUTE OF TECHNOLOGY \\ UNIVERSITY OF CALIFORNIA \\ MONTANA STATE UNIVERSITY \\ UNIVERSITY OF NEVADA \\ OREGON STATE COLLEGE \\ UNIVERSITY OF OREGON \\ OSAKA UNIVERSITY \\ UNIVERSITY OF SOUTHERN CALIFORNIA
}

\author{
STANFORD UNIVERSITY \\ UNIVERSITY OF TOKYO \\ UNIVERSITY OF UTAH \\ WASHINGTON STATE COLLEGE \\ UNIVERSITY OF WASHINGTON \\ * * * \\ AMERICAN MATHEMATICAL SOCIETY \\ CALIFORNIA RESEARCH CORPORATION \\ HUGHES AIRCRAFT COMPANY \\ SPACE TECHNOLOGY LABORATORIES
}

Mathematical papers intended for publication in the Pacific Journal of Mathematics should be typewritten (double spaced), and the author should keep a complete copy. Manuscripts may be sent to any one of the four editors. All other communications to the editors should be addressed to the managing editor, L. J. Paige at the University of California, Los Angeles 24, California.

50 reprints per author of each article are furnished free of charge; additional copies may be obtained at cost in multiples of 50 .

The Pacific Journal of Mathematics is published quarterly, in March, June, September, and December. The price per volume (4 numbers) is $\$ 12.00$; single issues, $\$ 3.50$. Back numbers are available. Special price to individual faculty members of supporting institutions and to individual members of the American Mathematical Society: $\$ 4.00$ per volume; single issues, $\$ 1.25$.

Subscriptions, orders for back numbers, and changes of address should be sent to Pacific Journal of Mathematics, 2120 Oxford Street, Berkeley 4, California.

Printed at Kokusai Bunken Insatsusha (International Academic Printing Co., Ltd.), No. 6, 2-chome, Fujimi-cho, Chiyoda-ku, Tokyo, Japan.

PUBLISHED BY PACIFIC JOURNAL OF MATHEMATICS, A NON-PROFIT CORPORATION

The Supporting Institutions listed above contribute to the cost of publication of this Journal, but they are not owners or publishers and have no responsibility for its content or policies. 


\section{Pacific Journal of Mathematics}

\section{Vol. 9, No. $2 \quad$ June, 1959}

Lee William Anderson, On the breadth and co-dimension of a topological lattice

Frank W. Anderson and Robert L. Blair, Characterizations of certain lattices

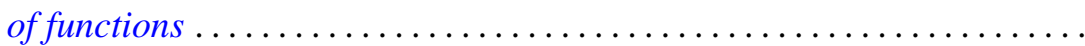

Donald Charles Benson, Extensions of a theorem of Loewner on integral

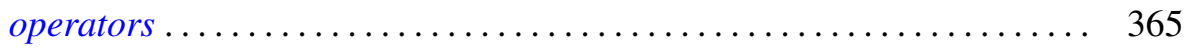

Errett Albert Bishop, A duality theorem for an arbitrary operator ........ 379

Robert McCallum Blumenthal and Ronald Kay Getoor, The asymptotic distribution of the eigenvalues for a class of Markov operators ........

Delmar L. Boyer and Elbert A. Walker, Almost locally pure Abelian

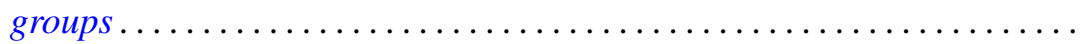

Paul Civin and Bertram Yood, Involutions on Banach algebras ........... Lincoln Kearney Durst, Exceptional real Lehmer sequences .... 415

Eldon Dyer and Allen Lowell Shields, Connectivity of topological

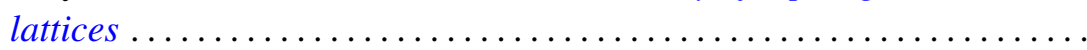

Ronald Kay Getoor, Markov operators and their associated

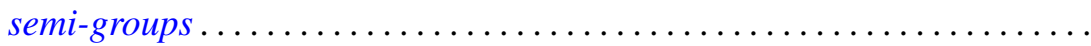

Bernard Greenspan, A bound for the orders of the components of a system of algebraic difference equations

Branko Grünbaum, On some covering and intersection properties in

Minkowski spaces ............................

Bruno Harris, Derivations of Jordan algebras ..............

Henry Berge Helson, Conjugate series in several variables.

Isidore Isaac Hirschman, Jr., A maximal problem in harmonic analysis.

II .

Alfred Horn and Robert Steinberg, Eigenvalues of the unitary part of a matrix

Edith Hirsch Luchins, On strictly semi-simple Banach algebras ...

William D. Munro, Some iterative methods for determining zeros of

functions of a complex variable...

John Rainwater, Spaces whose finest uniformity is metric .

William T. Reid, Variational aspects of generalized convex functions ....

A. Sade, Isomorphisme d'hypergroupoï des isotopes ...... . .

Isadore Manual Singer, The geometric interpretation of a special

connection . . .

Charles Andrew Swanson, Asymptotic perturbation series for characteristic

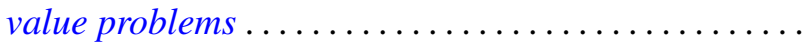

